\title{
Insegurança alimentar e nutricional de famílias usuárias da Estratégia Saúde da Família no interior da Paraíba
}

\section{Food and nutritional insecurity of families using the Family Health Strategy in the inner Paraíba State}

\author{
Eduarda Emanuela Silva dos Santos' (D), Ítalo de Macedo Bernardino' (D), \\ Dixis Figueroa Pedraza ${ }^{1,2}$ (D) \\ 'Universidade Estadual da Paraíba (UEPB) - Campina Grande (PB), Brasil. \\ 2Programa de Pós-graduação em Saúde da Família, Rede Nordeste de Formação em Saúde da Família, Brasil.
}

Como citar: Santos EES, Bernardino ÍM, Figueroa Pedrza D. Insegurança alimentar e nutricional de famílias usuárias da Estratégia Saúde da Família no interior da Paraíba. Cad Saúde Colet, 2021;29(1):110-121. https://doi.org/10.1590/1414-462X202129010412

\section{Resumo}

Introdução: A segurança alimentar e a melhoria da nutrição constituem um aspecto importante no desenvolvimento sustentável. Objetivo: Identificar fatores domiciliares que possam influenciar na segurança alimentar e nutricional de famílias com crianças menores de 5 anos em áreas de abrangência da Estratégia Saúde da Família (ESF). Método:Trata-se de um estudo transversal que envolveu 469 famílias de sete municípios do interior da Paraíba. Para a avaliação da segurança alimentar e nutricional das famílias foi utilizada a Escala Brasileira de Insegurança Alimentar (EBIA). Foram analisadas informações relacionadas aos determinantes domiciliares de insegurança alimentar moderada/grave. Resultados: A Insegurança Alimentar e Nutricional (IAN) atingiu 74,2\% das famílias, sendo 17,5\% de insegurança alimentar moderada/ grave. A variável que mais interferiu na insegurança alimentar moderada/grave foi maior vulnerabilidade socioeconômica. A não suplementação com ferro/ácido fólico durante a gravidez e recebimento do benefício do Programa Bolsa Família também representaram exposições associadas ao desfecho. Conclusão: Os resultados mostram prevalências elevadas de IAN, cujos graus mais graves sugerem a importância de melhorias na condição socioeconômica da família e o reforço das ações no contexto do Programa Bolsa Família e dos cuidados nutricionais durante a gravidez.

Palavras-chave: atenção primária à saúde; criança; segurança alimentar e nutricional; fatores socioeconômicos; programas governamentais.

\begin{abstract}
Background: Food security and improved nutrition are an important aspect of sustainable development. Objective: To identify household factors that may influence the food and nutritional security of families with children under five years of age in areas covered by the Family Health Strategy. Method: A crosssectional study involving 469 families from seven municipalities in the interior of Paraíba. The food and nutritional security of the families-was evaluated using the Brazilian Scale of Food Insecurity. We analyzed information related to household determinants of moderate/severe food insecurity. Results: Food and nutritional insecurity reached $74.2 \%$ of families, of which $17.5 \%$ were moderate/severe food insecurity. Higher socioeconomic vulnerability was the most interfered variable in moderate/severe food insecurity. Failure to supplement with iron/folic acid during pregnancy and to be beneficiaries of the Programa Bolsa Familia also represented exposures associated with the outcome. Conclusion: The results show high prevalence of food and nutritional insecurity, the most serious of which suggests the importance of
\end{abstract}

Trabalho realizado em Municípios do interior da Paraíba (PB), Brasil.

Correspondência: Eduarda Emanuela Silva dos Santos. E-mail: dudanutri@hotmail.com

Fonte de financiamento: nenhuma.

Conflito de interesses: nada a declarar.

Recebido em: Set. 23, 2019. Aceito em: Fev. 19, 2020
Este é um artigo publicado em acesso aberto (Open Access) sob a licença Creative Commons Attribution, que permite uso, distribuição e reprodução em qualquer meio, sem restrições desde que o trabalho original seja corretamente citado. 
improvements in the socioeconomic status of the family and the reinforcement of actions in the context of the Programa Bolsa Família and nutritional care during pregnancy.

Keywords: primary health care; child; food and nutrition security; socioeconomic factors; government programs.

\section{INTRODUÇÃO}

A Insegurança Alimentar e Nutricional (IAN) é altamente prevalente nos países em desenvolvimento, sendo reconhecida como sério problema de saúde pública'. Estima-se que no mundo aproximadamente 821 milhões de pessoas, cerca de uma em cada nove, sejam incapazes de satisfazer suas necessidades básicas de alimentos. De forma similar à prevalência de subalimentação, a insegurança alimentar grave cresce ao nível mundial, impulsionado pelas tendências observadas na África e América Latina onde as prevalências aumentaram de 22,3\% e 7,6\% em 2014 para $29,8 \%$ e 9,8\% em 2017, respectivamente 2 . No Brasil, 22,6\% dos domicílios apresentam algum grau de IAN, sendo 3,2\% grave ${ }^{3}$. Nessa conjuntura, acabar com a fome, alcançar a segurança alimentar e melhoria da nutrição e promover a agricultura sustentável constitui um dos objetivos globais do desenvolvimento sustentável ${ }^{4}$. O Brasil, como signatário da Agenda 2030 para o Desenvolvimento Sustentável, é um dos poucos países que dispõe de um instrumento que orienta a territorialização desses objetivos ${ }^{5}$.

No contexto familiar, a IAN manifesta-se de forma progressiva, contemplando desde a preocupação quanto à disponibilidade futura de comida até a ocorrência da fome ${ }^{6}$. Dessa forma, nas suas diversas perspectivas e caráter interdisciplinar, a Segurança Alimentar e Nutricional (SAN) no domicílio relaciona-se diretamente às determinantes domiciliares que são influenciados pelas condições macrossocioeconômicas e regionais-locais. Características como o perfil demográfico e de saúde, a renda/estabilidade financeira, a escolaridade, a raça/ cor, o emprego e o tempo disponível da mãe, a educação alimentar, o comportamento e os hábitos alimentares, a rede social e a participação em programas assistenciais são determinantes domiciliares da IAN. O conhecimento da associação desses fatores com a situação de SAN das famílias é essencial à proposição de ações direcionadas a garantir o direito humano à alimentação ${ }^{7}$.

Nesse sentido, pesquisas têm mostrado importantes desigualdades que permeiam a situação de IAN entre as famílias brasileiras, com prevalências maiores na região Nordeste ${ }^{3}$, nos estratos de maior vulnerabilidade social, econômica e demográfica ${ }^{8}$, em famílias que utilizam serviços públicos de saúde/beneficiários do Programa Bolsa Família $(\mathrm{PBF})^{9}$ e em domicílios com crianças menores de 5 anos de idade ${ }^{10}$. Entretanto, até o momento nenhum estudo foi publicado com base em análises através de modelos estatísticos sob a ótica de suporte à tomada de decisão em saúde ${ }^{11}$. Análises desse tipo podem ser relevantes à compreensão da IAN como fenômeno complexo e multidimensional de determinação multicausal, além da sua determinação social conforme evidenciado em revisão sistemática da literatura ${ }^{9}$. Sendo assim, o presente artigo justifica-se pela importância de identificar fatores domiciliares que possam influenciar a SAN de famílias com crianças menores de 5 anos de áreas de abrangência da Estratégia Saúde da Família (ESF).

\section{MÉTODO}

Trata-se de um estudo transversal realizado com famílias residentes em municípios do Estado da Paraíba, atendidas na ESF, com crianças menores de 5 anos no núcleo familiar. A pesquisa foi desenvolvida em sete municípios do interior do Estado, com população entre 30.000 e 149.999 habitantes, que recebem incentivos de custeio para a implementação de ações de prevenção e controle do sobrepeso em crianças $^{12}$. Do total de 12 municípios com o benefício, cinco foram excluídos: um por ser o único com cobertura parcial da ESF, outro devido a não possuir equipes de saúde predeterminadas como de interesse para o estudo (equipes convencionais vinculadas a Núcleo de Apoio à Saúde da Família sem atuação de nutricionista e equipes do Programa Mais Médicos vinculadas a Núcleo de Apoio à Saúde da Família com atuação de nutricionista) e três considerando sua inserção em outra proposta 
com características similares. Participaram do estudo 22 equipes de saúde da ESF e 11 creches vinculadas às mesmas no contexto do Programa Saúde na Escola.

Para a definição da amostra, foi utilizada a prevalência de IAN moderada/grave de 31,6\% em famílias que usam serviços públicos de saúde/beneficiárias do $\mathrm{PBF}^{9}$. Para o cálculo foi utilizada a fórmula $\mathrm{n}=[\mathrm{N} . Z 2 . p .(1-\mathrm{p})] /$ [Z2.p. $(1-\mathrm{p})+\mathrm{e}(\mathrm{N}-1)]$. Considerando $\mathrm{N}=23.089$, como erro amostral máximo de $5 \%$ sob nível de significância de $95 \%$ e $30 \%$ para compensar eventuais perdas e controle de fatores de confusão, estimou-se a necessidade de estudar 469 famílias. Essa amostra, apesar de perdas ocorridas, ficou inalterada, pois a decisão inicial dos gestores de um município em não participar na pesquisa foi mudada em seu decorrer e solicitada sua inclusão. Assim, os pesquisadores, pautados principalmente em aspectos éticos, decidiram pela coleta de dados no município em questão até completar a amostra de estudo.

A quantidade de equipes de saúde e de creches foi determinada proporcionalmente ao número de famílias residentes com crianças menores de 5 anos, contemplando-se tanto equipes de saúde convencionais quanto do Programa Mais Médicos, respeitando a composição e o mínimo de uma creche por localidade. O número de famílias em cada município também foi estabelecido de forma proporcional à quantidade de domicílios com crianças menores de 5 anos. Não foi instituído um número a priori para cada equipe de saúde. Para as creches, o quantitativo de famílias foi definido de forma que permitisse completar o total previsto por município, considerando as observações realizadas no contexto das equipes de saúde.

A amostra foi selecionada em dois estágios. No primeiro, foram sorteadas aleatoriamente as equipes de saúde e creches, e no segundo estágio as famílias. Para as equipes de saúde, as famílias foram, primeiramente, selecionadas de forma aleatória por ocasião do atendimento de puericultura da criança no dia de coleta de dados. Em um segundo momento, realizou-se busca ativa das famílias, com base nos registros das equipes de saúde, sendo a seleção por sorteio aleatório. Nas creches, a seleção probabilística das famílias foi a partir das listas das crianças disponíveis nessas instituições, considerando-se como uma unidade amostral os casos de irmãos na mesma creche e excluindo-se os ausentes no dia do trabalho de campo. A coleta de dados foi realizada nas unidades de saúde e creches no segundo semestre de 2018 por profissionais e estudantes capacitados e supervisionados.

O instrumento de coleta incluiu a avaliação da SAN familiar (variável dependente) e a aplicação de um questionário estruturado às mães das crianças contendo informações relacionadas aos seus determinantes domiciliares, segundo o modelo proposto por Kepple e Segall-Corrêa ${ }^{7}$. Assim, obtiveram-se informações sobre o perfil demográfico da criança (sexo e idade); a saúde materno-infantil (características da gravidez em relação ao consumo de álcool e de cigarro, suplementação de ferro/ácido fólico, vacinação antitetânica e desenvolvimento de complicações clínicas, e hospitalização da criança nos últimos 12 meses); a rede social (convivência da mãe com companheiro e apoio social); a participação em programas assistenciais (frequência da criança à creche e benefício do PBF) e a situação socioeconômica da família (trabalho materno fora de casa e classificação socioeconômica).

O apoio social foi avaliado por meio do questionário do Medical Outcomes Study. Esse instrumento é composto por 19 itens em cinco dimensões: material, afetiva, emocional, informação e interação social. Para todas as perguntas, são cinco as opções de respostas (sempre, que equivale a cinco pontos; quase sempre, a quatro pontos; às vezes, a três pontos; raramente, a dois pontos; e nunca). Em cada uma das dimensões, os escores dos itens foram adicionados; o escore total foi dividido pelo escore máximo possível para a dimensão e multiplicado por $100^{13,14}$. Os escores foram dicotomizados com base na mediana mais frequentemente encontrada nas análises das diferentes dimensões de apoio social. Dessa forma, utilizou-se como ponto de corte único o "escore $>90$ " como indicativo de mais altos níveis de apoio social.

A classificação socioeconômica da família baseou-se nos critérios da Associação Brasileira de Empresas de Pesquisa ${ }^{15}$, que é usado para estimar o poder de compra de famílias brasileiras. Para esta classificação, considera-se a existência de vaso sanitário no domicílio; a contratação de empregada doméstica; a posse de bens; o grau de instrução do chefe de família e o acesso a serviços públicos. As famílias foram classificadas nas classes $\mathrm{A} / \mathrm{B} / \mathrm{C}$ ou $\mathrm{D} / \mathrm{E}$. 
A situação de SAN das famílias foi avaliada por meio da Escala Brasileira de Insegurança Alimentar (EBIA) com 14 itens 6 . Trata-se é uma escala validada que possibilita classificar as famílias em diferentes graus de IAN: segurança quando todas as questões são respondidas de forma negativa, insegurança leve quando se tem até cinco questões com respostas positivas, insegurança moderada nos casos de seis a nove respostas positivas e insegurança grave para 10 a 14 respostas positivas.

O grupo de variáveis independentes foi formado pelas seguintes características: perfil demográfico da criança (idade, sexo); perfil de saúde materno-infantil (as seguintes características durante a gestação são observadas, como: o consumo de álcool e de cigarro, suplementação de ferro/ácido fólico, vacinação antitetânica, complicação clínica e a hospitalização da criança nos últimos 12 meses); rede social (convivência da mãe com companheiro e apoio social); participação em programas assistenciais (frequência da criança à creche e benefício do PBF) e situação socioeconômica da família (trabalho materno fora de casa e classificação socioeconômica). A SAN foi a variável dependente do estudo, dicotomizada em segurança alimentar/insegurança alimentar leve e insegurança alimentar moderada/grave.

Os dados coletados foram organizados em planilhas eletrônicas e digitados em dupla entrada em um banco de dados customizado com verificações de consistência e restrições de intervalo. Utilizou-se para as análises estatísticas o banco produzido após verificação de análise de consistência dos dados digitados.

Inicialmente, foram calculadas as frequências absolutas e percentuais de todas as variáveis em estudo. Em seguida, foi realizada a análise bivariada para testar a associação entre o nível de SAN e todas as variáveis independentes, utilizando o Teste Qui-quadrado. Para explicar a insegurança alimentar moderada/grave, inseriram-se todas as variáveis em um modelo de Árvore de Decisão por meio do algoritmo CHAID.

A Árvore de Decisão consiste em regras de decisão que realizam sucessivas divisões no conjunto de dados, de modo a torná-lo cada vez mais homogêneo no tocante à variável dependente. A técnica permite identificar as variáveis mais relevantes na descrição de um problema e suas relações hierárquicas. Para esses fins, os componentes do problema são dispostos de forma gráfica, permitindo segmentar a população em subconjuntos significativos que podem representar o foco de intervenções. Dessa forma, possibilita inferências que não são admissíveis em outros modelos como a regressão em que as decisões são voltadas para o membro médio da população, sem discriminar subgrupos populacionais ${ }^{16,17}$.

A Árvore de Decisão foi construída utilizando um gráfico que começa com um nó raiz, em que todas as observações da amostra são apresentadas. Os nós produzidos em sequência representam subdivisões dos dados em grupos cada vez mais homogêneos, sendo denominados de nós-filhos. Quando não há mais possibilidade de divisão, os nós são chamados de nós-terminais ou folhas ${ }^{18}$. $\mathrm{O}$ modelo foi ajustado mediante sucessivas divisões binárias (nós) nos conjuntos de dados. O critério de parada adotado foi o valor $p<0,05$ da estatística $X^{2}$ usando a correção de Bonferroni. $O$ ajuste do modelo final foi avaliado pela estimativa de risco geral, que compara a diferença entre o valor esperado e o observado, indicando em que medida a árvore corretamente prediz os resultados ${ }^{19}$. Só permaneceram no gráfico final as variáveis que obtiveram valor $p$ ajustado $<0,05$.

As análises estatísticas foram feitas no programa SPSS (Statistical Package for Social Sciences) versão 20.0 (SPSS Inc., Chicago, Estados Unidos).

O projeto foi aprovado pelo Comitê de Ética em Pesquisa da Universidade Estadual da Paraíba (CAAE 71609417.7.0000.5187). Todos os participantes da pesquisa assinaram o Termo de Consentimento Livre e Esclarecido.

\section{RESULTADOS}

Participaram do estudo 469 famílias com crianças menores de 5 anos. De acordo com a EBIA, 56,7\% das famílias apresentaram algum grau de IAN, sendo moderada/grave em 17,5\% dos domicílios (Figura 1). 


\section{Nível de Segurança Alimentar e Nutricional}

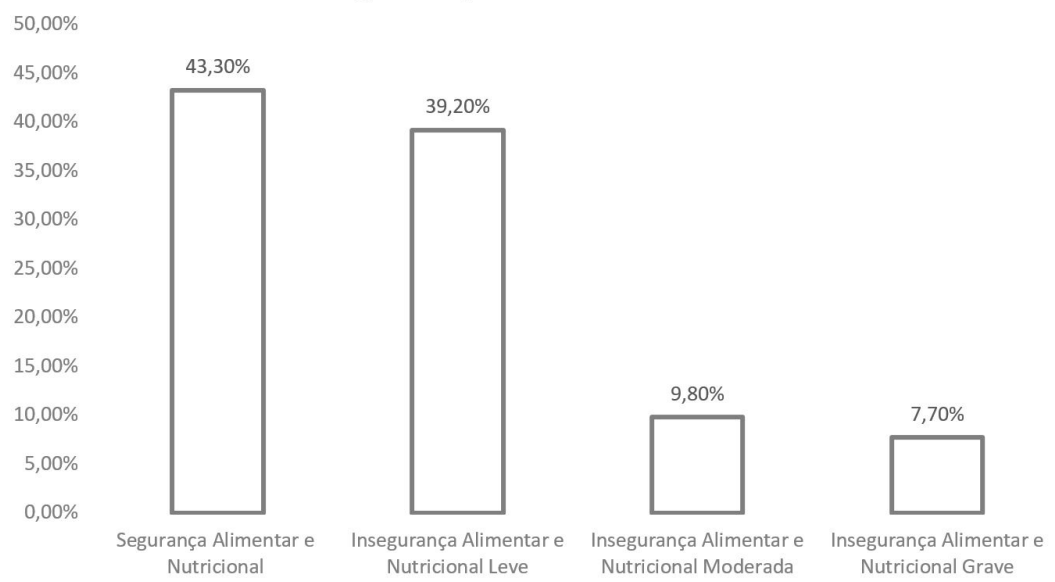

Figura 1. Prevalências de Segurança Alimentar e Nutricional em famílias com crianças menores de cinco anos residentes em áreas de abrangência da Estratégia Saúde da Família do Estado da Paraíba, 2018

Do total de famílias, $243(51,8 \%)$ eram formadas por crianças do sexo masculino e 252 $(53,7 \%)$ de crianças maiores de 2 anos. No perfil de saúde materno-infantil, destaca-se que $71,9 \%$ das mães tiveram alguma complicação clínica durante a gestação e $84,4 \%$ das crianças foram internadas nos últimos 12 meses. A maioria das famílias participava do PBF $(73,8 \%)$ e foi classificada nas classes socioeconômicas D/E (Tabela 1).

Em relação às análises bivariadas (Tabela 1), observou-se que o consumo de álcool, de cigarro e a não suplementação de ferro/ácido fólico durante a gravidez representaram exposições associadas à insegurança alimentar moderada/grave. Além disso, a não convivência da mãe com companheiro $(p=0,010)$, o baixo apoio social $(p=0,009)$, participar do PBF $(p<$ $0,001)$ e pertencer às classes socioeconômicas $D / E(p<0,001)$ também expressaram valores significativos de associação.

O modelo final da Árvore de Decisão foi construído por sete nós, conforme mostrado na Figura 2. Segundo o modelo, a variável que mais interferiu na presença de insegurança alimentar moderada/grave foi o nível socioeconômico $(p<0,001)$, sendo observada prevalência de $7,1 \%$ do desfecho entre as famílias das classes $A / B / C$ e de $23,3 \%$ nos casos das classes D/E. Para as famílias de melhor condição socioeconômica, ser beneficiário do PBF representou uma exposição importante na insegurança alimentar moderada/grave $(p=0,004)$. No entanto, nos domicílios mais afetados, ou seja, das classes socioeconômicas inferiores, a não suplementação durante a gravidez expressou sua importância na insegurança alimentar moderada/grave com prevalência de $45,0 \%(p=0,018)$.

\section{DISCUSSÃO}

Com o objetivo de identificar fatores domiciliares que possam influenciar a IAN de famílias com crianças menores de 5 anos de áreas de abrangência da ESF, este estudo utilizou um modelo multivariado de árvore de decisão. Sua estrutura é composta de nós, ramos e desfechos, permitindo a sistematização dos dados de modo a auxiliar o processo de tomada de decisão e contribuir para alocação eficiente dos recursos ${ }^{16,19}$. Nesse sentido, os achados reforçam a importância da situação socioeconômica da família e da participação em programas sociais em casos de vulnerabilidade na IAN, ficando implícita a necessidade de apreciar esses fatores tanto na análise das probabilidades de uma família estar com dificuldades de acesso à alimentação quanto no fortalecimento das políticas de SAN.

A prevalência de IAN $(56,7 \%)$ na população estudada foi maior que a reportada nacionalmente na Pesquisa Nacional de Demografia e Saúde $(37,5 \%)^{20}$ e na Pesquisa Nacional por Amostra de Domicílios $(22,6 \%)^{3}$. Entretanto, a prevalência encontrada é mais próxima da estimada para domicílios brasileiros com crianças menores de 5 anos $(45,4 \%)^{10}$ e também 
Tabela 1. Situação de Segurança Alimentar e Nutricional, de acordo com determinantes domiciliares (perfil demográfico da criança, perfil de saúde materno-infantil, rede social, participação em programas assistenciais e situação socioeconômica), em famílias com crianças menores de cinco anos de áreas de abrangência da Estratégia Saúde da Família do Estado da Paraíba, 2018

\begin{tabular}{|c|c|c|c|c|}
\hline \multirow{3}{*}{ Variáveis } & \multirow{3}{*}{$\begin{array}{l}\text { Total } \\
\text { n (\%) }\end{array}$} & \multicolumn{2}{|c|}{ Segurança Alimentar e Nutricional } & \multirow{3}{*}{ p-valor } \\
\hline & & \multirow{2}{*}{$\begin{array}{c}\text { Nível A } \\
\mathbf{n}(\%)\end{array}$} & \multirow{2}{*}{$\begin{array}{c}\text { Nível B } \\
\text { n (\%) }\end{array}$} & \\
\hline & & & & \\
\hline \multicolumn{5}{|c|}{ Perfil demográfico da criança } \\
\hline \multicolumn{2}{|l|}{ Idade (anos) } & & & 0,796 \\
\hline$\geq 2$ & $252(53,7)$ & $209(82,9)$ & $43(17,1)$ & \\
\hline$<2$ & $217(46,3)$ & $178(82,0)$ & $39(18,0)$ & \\
\hline \multicolumn{2}{|l|}{ Sexo } & & & 0,718 \\
\hline Masculino & $243(51,8)$ & $202(83,1)$ & $41(16,9)$ & \\
\hline Feminino & $226(48,2)$ & $185(81,9)$ & $41(18,1)$ & \\
\hline \multicolumn{5}{|c|}{ Perfil de saúde materno-infantil } \\
\hline \multicolumn{3}{|c|}{ Consumo de álcool na gravidez } & & 0,003 \\
\hline Não & $420(89,5)$ & $354(84,3)$ & $66(15,7)$ & \\
\hline Sim & $49(10,5)$ & $33(67,3)$ & $16(32,7)$ & \\
\hline \multicolumn{3}{|c|}{ Consumo de cigarro na gravidez } & & 0,009 \\
\hline Não & $437(93,2)$ & $366(83,8)$ & $71(16,2)$ & \\
\hline Sim & $32(6,8)$ & $21(65,6)$ & $11(34,4)$ & \\
\hline \multicolumn{4}{|c|}{ Suplementação de ferro/ácido fólico na gravidez } & 0,018 \\
\hline Sim & $443(94,5)$ & $370(83,5)$ & $73(16,5)$ & \\
\hline Não & $26(5,5)$ & $17(65,4)$ & $9(34,6)$ & \\
\hline \multicolumn{3}{|c|}{ Vacinação antitetânica na gravidez } & & 0,709 \\
\hline Sim & $428(91,6)$ & $352(82,2)$ & $76(17,8)$ & \\
\hline Não & $39(8,4)$ & $33(84,6)$ & $6(15,4)$ & \\
\hline \multicolumn{3}{|c|}{ Complicação clínica durante a gestação } & & 0,430 \\
\hline Não & $132(28,1)$ & $106(80,3)$ & $26(19,7)$ & \\
\hline Sim & $337(71,9)$ & $281(83,4)$ & $56(16,6)$ & \\
\hline \multicolumn{4}{|c|}{ Hospitalização da criança nos últimos 12 meses } & 0,079 \\
\hline Não & $73(15,6)$ & $55(75,3)$ & $18(24,7)$ & \\
\hline Sim & $396(84,4)$ & $332(83,8)$ & $64(16,2)$ & \\
\hline \multicolumn{5}{|l|}{ Rede social } \\
\hline \multicolumn{3}{|c|}{ Convivência da mãe com companheiro } & & 0,010 \\
\hline Sim & $350(74,6)$ & $298(85,1)$ & $52(14,9)$ & \\
\hline
\end{tabular}

Nível A: Segurança alimentar/insegurança alimentar leve, Nível B: Insegurança alimentar moderada/grave, n: número absoluto, $p$-valor: valor de significância estatística segundo o Teste Qui-quadrado; valor de $\mathrm{p}<0,05$ 
Tabela 1. Continuação...

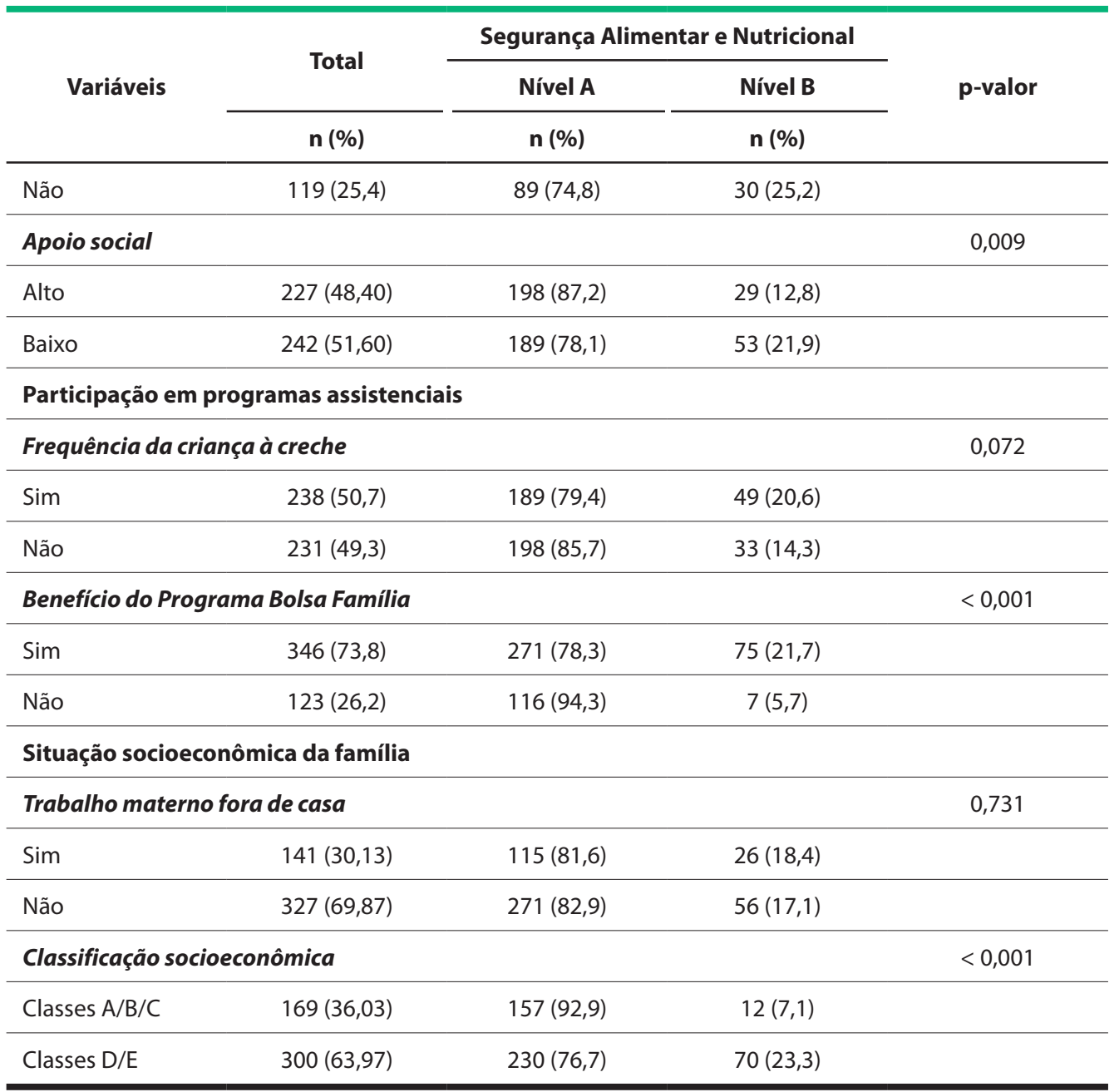

Nível A: Segurança alimentar/insegurança alimentar leve, Nível B: Insegurança alimentar moderada/grave, n: número absoluto, p-valor: valor de significância estatística segundo o Teste Qui-quadrado; valor de $\mathrm{p}<0,05$

dos valores encontrados em famílias residentes no município de Campina Grande, PB, com crianças na mesma faixa etária $(69,2 \%)^{21}$. Isso parece não ser apenas um problema no Brasil, revelando-se, por exemplo, também alta prevalência de IAN entre os menores de 5 anos no México $(75,7 \%)^{22}$ e na província costeira do Quênia $(81,9 \%)^{23}$. Tal fato reforça o contexto de vulnerabilidade apresentado pelas famílias estudadas, demonstrado pelos baixos níveis econômicos e de inserção da mulher no mercado de trabalho, assim como grande participação em programas sociais de transferência de renda.

Um ponto importante a se destacar é que famílias residentes em áreas de abrangência da ESF podem ter maiores chances de IAN, uma vez que as condições de vida enfrentadas por essa população, que em sua maioria apresenta maior vulnerabilidade socioeconômica, prejudica o acesso aos alimentos ${ }^{9}$. Crianças como as do presente estudo, residentes de domicílios com cobertura da ESF, vivem em contextos de frágil infraestrutura sanitária, social e de lazer, assim como têm piores condições de saúde ${ }^{24}$, que podem influenciar negativamente sua SAN7. A área estudada compreendeu sete municípios nordestinos com Índice de Desenvolvimento Humano Municipal (IDHM) baixo e médio, com componentes mais precários para a renda e educação, e circunstâncias sanitárias desfavoráveis que podem influenciar nas condições inadequadas de vida e alimentação. 
NÍVEL DE SEGURANÇA ALIMENTAR E NUTRICIONAL

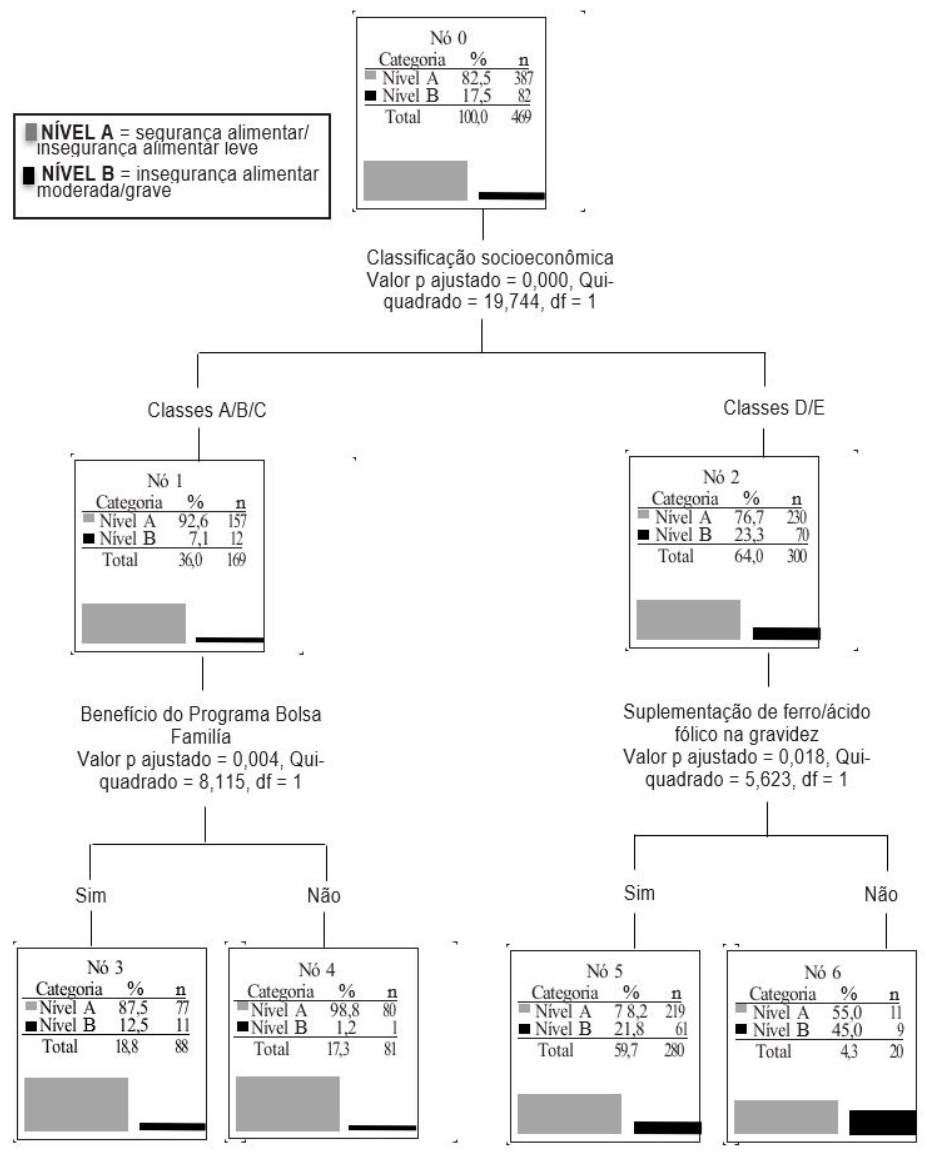

Figura 2. Análise multivariada por meio da Árvore de Decisão para a insegurança alimentar moderada/ grave, ajustada pelos fatores investigados, em famílias com crianças menores de cinco anos de áreas de abrangência da Estratégia Saúde da Família do estado da Paraíba, 2018. Df: degrees of freedom

Considerando que a insegurança alimentar moderada/grave é uma situação familiar de privação de alimentos, registra-se que 17,5\% dos domicílios com crianças menores de 5 anos encontravam-se nessa situação, segundo os resultados apresentados. Prevalências semelhantes foram registradas por outros pesquisadores, incluindo análises de âmbito nacional ${ }^{10}$, estadual ${ }^{3}$ e municipal ${ }^{25}$. Entretanto, outros estudos de abrangência local têm mostrado prevalências maiores ${ }^{21,26,27}$. As diferenças entre as prevalências de IAN podem ser interpretadas como reflexo da heterogeneidade na sua distribuição geográfica, como a alta variação de insegurança alimentar grave entre os municípios da Região Nordeste, de $0,83 \%$ a $18,58 \%{ }^{28}$. A ocorrência da IAN ligada a determinantes econômicos e sociais explica tais desigualdades, devendo ser consideradas nas ações de combate à fome ${ }^{8,28}$.

Na Árvore de Decisão foi possível observar que as maiores prevalências de insegurança alimentar moderada/grave, constatadas entre as famílias das classes socioeconômicas inferiores, confirmam os resultados obtidos tanto em pesquisas de âmbito nacional ${ }^{10,29} \mathrm{como} \mathrm{local}^{21,26}$. A sistematização de estudos sobre o tema também tem permitido obter achados nesse sentido, destacando-se a IAN condicionada a fatores como baixa renda, ausência de vínculo empregatício, baixa escolaridade, moradia inadequada e ausência de saneamento básico ${ }^{9,30}$. No Brasil, a renda insuficiente, por implicar em dificuldade de acesso regular e permanente aos alimentos, tem sido descrita como um dos determinantes mais importantes da IAN ${ }^{31}$, fato que revela consistência em relação ao comportamento da variável que representou o nó raiz na Árvore de Decisão. Esses achados são preocupantes, uma vez que crianças que crescem 
em famílias socialmente vulneráveis, inclusive de IAN, apresentam piores condições de saúde na infância e capacidade produtiva reduzida na vida adulta que pode perpetuar-se na forma de um ciclo intergeracional ${ }^{10,32}$.

Neste estudo, a maior probabilidade de insegurança alimentar moderada/grave nas famílias beneficiárias do PBF, entre aquelas de melhor condição socioeconômica, segue a tendência mostrada em domicílios urbanos com crianças de áreas de abrangência de unidades básicas de saúde ${ }^{29,33}$. Essa perspectiva é ampliada através de revisões da literatura que destacaram a impossibilidade do PBF resolver apenas com suas ações o problema da $I_{A N} \mathrm{~N}^{31,34}$ e a maior chance desse desfecho entre usuários do Programa e/ou de serviços de saúde, em comparação com populações de referência ${ }^{9}$. Tais achados sugerem o adequado direcionamento dos recursos do Programa, entretanto sem conseguir atingir a SAN, reforçando a influência predominante da renda per capita na IAN e a necessidade de aumento dos repasses financeiros em consonância com a realidade e os impactos desejados ${ }^{21,35}$. Adicionalmente, é importante salientar a importância de associar o benefício monetário a outros tipos de intervenções como ações de educação nutricional, suplementação, fortificação, alimentação escolar, apoio à agricultura familiar, estabilidade dos preços e disponibilidade de serviços de saúde de qualidade que contribuam no cumprimento das condicionalidades de saúde e no uso adequado das transferências monetárias ${ }^{31,34}$.

Em classes socioeconômicas inferiores, a maior probabilidade de insegurança alimentar moderada/grave, entre mães que não receberam suplementação durante a gravidez, segue a tendência mostrada em estudo prévio no qual os cuidados inadequados na gestação, incluído a suplementação com micronutrientes, representou maiores chances de IAN em graus mais severos ${ }^{27}$. Os efeitos da IAN podem ser vistos principalmente entre os grupos mais vulneráveis, nos quais a falta de alimentos saudáveis e de qualidade pode estar relacionada ao acesso precário a renda, bens e serviços ${ }^{36}$. A consistência desses achados também encontra explicações segundo uma revisão da literatura que destaca o nível socioeconômico como o principal determinante da suplementação com ácido fólico na gestação ${ }^{37}$, sendo que a IAN medida por meio da EBIA é considerada um importante indicador de iniquidade social ${ }^{38}$. Além disso, destaca-se que a melhor situação socioeconômica pode favorecer o acesso e a utilização de serviços e tecnologias de melhor qualidade ${ }^{39}$. Portanto, é preciso que os profissionais das unidades básicas de saúde direcionem atenção às mulheres em situação de IAN e que não recebem a suplementação, para prevenir a anemia na gestação e promover a saúde maternoinfantil ${ }^{40}$.

Como é próprio de estudos transversais, as limitações do deste artigo incluem a impossibilidade de estabelecer relações causais entre as variáveis analisadas. Além disso, por conta da EBIA basear-se na percepção e nas diferentes experiências dos entrevistados, não deve ser excluída a influência do viés de memória e de uma informação diferencial de IAN. Em contrapartida, o estudo permitiu obter prevalências e determinar associações desagregadas sobre a IAN de um subgrupo da população, de grande utilidade para a identificação de vulnerabilidades que são relevantes ao planejamento ou reformulação de políticas públicas destinadas à solução do problema.

Os resultados do presente estudo mostram que, entre cada dez famílias com crianças menores de 5 anos usuárias da ESF de municípios do interior da Paraíba, cerca de seis e duas vivem em estado de IAN e insegurança alimentar moderada/grave, respectivamente; taxas que podem ser consideradas elevadas. Entre essas famílias, a insegurança alimentar moderada/ grave relaciona-se de maneira mais relevante com a condição socioeconômica, indicando que a redução quantitativa e/ou qualitativa da alimentação, associada à menor capacidade aquisitiva, constitui um aspecto essencial que deve ser melhorado tanto no seio da família quanto do ponto de vista de políticas públicas. Além disso, distinguem-se grupos homogêneos nos quais outros fatores também representam exposições de importância na situação de SAN, especificamente a não suplementação durante a gravidez e o benefício do PBF nas famílias de pior e melhor condição socioeconômica, respectivamente. 


\section{REFERÊNCIAS}

1. Motbainor A, Worku A, Kumie A. Level and determinants of food insecurity in East and West Gojjam zones of Amhara Region, Ethiopia: a community based comparative cross-sectional study. BMC Public Health. 2016;16(1):503. http://dx.doi.org/10.1186/s12889-016-3186-7. PMid:27289456.

2. Organización de las Naciones Unidas para la Alimentación y la Agricultura. Fondo Internacional de Desarrollo Agrícola. United Nations International Children's Emergency Fund. Programa Mundial de Alimentos. Organización Mundial de la Salud. El estado de la seguridad alimentaria y la nutrición en el mundo. Fomentando la resiliencia climática en aras de la seguridad alimentaria y la nutrición. Roma: FAO; 2018.

3. Instituto Brasileiro de Geografia e Estatística. Pesquisa Nacional por Amostra de Domicílios: segurança alimentar 2013. Rio de Janeiro: IBGE; 2014.

4. Organização das Nações Unidas. Transformando Nosso Mundo: a Agenda 2030 para o Desenvolvimento Sustentável [Internet]. Rio de Janeiro: Centro de Informação das Nações Unidas para o Brasil; 2016 [citado em 2019 abr 11]. Disponível em:https://www.mds.gov.br/webarquivos/publicacao/Brasil_Amigo_Pesso_ Idosa/Agenda2030.pdf

5. Instituto de Pesquisa Econômica Aplicada (IPEA). ODS - Metas Nacionais dos Objetivos de Desenvolvimento Sustentável. Brasília: IPEA; 2018.

6. Segall-Corrêa AM, Marin-León L, Melgar-Quiñonez H, Pérez-Escamilla R. Refinement of the Brazilian Household Food Insecurity Measurement Scale: Recommendation for a 14-item EBIA. Rev Nutr. 2014;27(2):241-51. http://dx.doi.org/10.1590/1415-52732014000200010.

7. Kepple AW, Segall-Corrêa AM. Conceituando e medindo segurança alimentar e nutricional. Cien Saude Colet. 2011;16(1):187-99. http://dx.doi.org/10.1590/S1413-81232011000100022. PMid:21180827.

8. Santos TG, Silveira JAC, Longo-Silva G, Ramires EKNM, Menezes RCE. Tendência e fatores associados à insegurança alimentar no Brasil: Pesquisa Nacional por Amostra de Domicílios 2004, 2009 e 2013. Cad Saude Publica. 2018;34(4):e00066917. http://dx.doi.org/10.1590/0102-311x00066917. PMid:29617484.

9. Bezerra TA, Olinda RA, Pedraza DF. Insegurança alimentar no Brasil segundo diferentes cenários sociodemográficos. Cien Saude Colet. 2017;22(2):637-51. http://dx.doi.org/10.1590/141381232017222.19952015.

10. Poblacion AP, Marín-León L, Segall-Corrêa NA, Silveira JAC, Taddei JAAC. Insegurança alimentar em domicílios brasileiros com crianças menores de cinco anos. Cad Saude Publica. 2014;30(5):1067-78. http:// dx.doi.org/10.1590/0102-311X00072713. PMid:24936822.

11. Moraes RM, Soares RAS. Modelos de Decisão aplicados à Saúde: teoria e prática. Tempus Actas Saúde Colet. 2016;10(2):7-10. http://dx.doi.org/10.18569/tempus.v10i2.1890.

12. Brasil. Portaria $n^{\circ} 2.706$, de 18 de outubro de 2017. Lista os municípios que finalizaram a adesão ao Programa Saúde na Escola para o ciclo 2017/2018 e os habilita ao recebimento do teto de recursos financeiros pactuados em Termo de Compromisso e repassa recursos financeiros para Municípios prioritários para ações de prevenção da obesidade infantil com escolares. Diário Oficial da União, Brasília, 20 de outubro de 2017.

13. Griep RH, Chor D, Faerstein E, Werneck GL, Lopes CS. Validade de constructo de escala de apoio social do Medical Outcomes Study adaptada para o português no Estudo Pró-Saúde. Cad Saude Publica. 2005;21(3):703-14. http://dx.doi.org/10.1590/S0102-311X2005000300004. PMid:15868028.

14. Chor D, Griep RH, Lopes CS, Faerstein E. Medidas de rede e apoio social no Estudo Pró-Saúde: prétestes e estudo piloto. Cad Saude Publica. 2001;17(4):887-96. http://dx.doi.org/10.1590/S0102311X2001000400022. PMid:11514869.

15. Associação Brasileira de Empresas de Pesquisa. Critério de classificação econômica Brasil. São Paulo: ABEP; 2016.

16. de Soárez PC, Soares MO, Novaes HM. Modelos de decisão para avaliações econômicas de tecnologias em saúde. Cien Saude Colet. 2014;19(10):4209-22. http://dx.doi.org/10.1590/1413-812320141910.02402013. PMid:25272129.

17. Speybroeck N. Classification and regression trees. Int J Public Health. 2012;57(1):243-6. http://dx.doi. org/10.1007/s00038-011-0315-z. PMid:22015650.

18. Hair JF Jr, Black WC, Babin BJ, Anderson RE, Tatham R. Análise multivariada de dados. 6a Edição. Porto Alegre: Bookman; 2009.

19. Bernardino IM, Barbosa KGN, Nóbrega LM, Cavalcante GMS, Ferreira EF, d'Avila S. Violência contra mulheres em diferentes estágios do ciclo de vida no Brasil: um estudo exploratório. Rev Bras Epidemiol. 2016;19(4):740-52. http://dx.doi.org/10.1590/1980-5497201600040005. PMid:28146164. 
20. Brasil. Ministério da Saúde. Pesquisa Nacional de Demografia e Saúde da Criança e da Mulher - PNDS 2006: dimensões do processo reprodutivo e da saúde da criança. Brasília: Ministério da Saúde; 2009.

21. Pedraza DF, Gama JS. Segurança alimentar e nutricional de famílias com crianças menores de cinco anos do município de Campina Grande, Paraíba. Rev Bras Epidemiol. 2015 out-dez;18(4):906-17. http://dx.doi. org/10.1590/1980-5497201500040018. PMid:26982304.

22. Cuevas-Nasu L, Rivera-Dommarco JA, Shamah-Levy T, Mundo-Rosas V, Méndez-Gómez Humarán I. Inseguridad alimentaria y estado de nutrición en menores de cinco años de edad en México. Salud Publica Mex. 2014;56(Supl. 1):s47-53. http://dx.doi.org/10.21149/spm.v56s1.5165. PMid:25649452.

23. Shinsugi C, Matsumura M, Karama M, Tanaka J, Changoma M, Kaneko S. Factors associated with stunting among children according to the level of food insecurity in the household: a cross-sectional study in a rural community of Southeastern Kenya. BMC Public Health. 2015;15(1):441. http://dx.doi.org/10.1186/ s12889-015-1802-6. PMid:25924925.

24. Oliveira BLCA, Moreira JPL, Luiz RR. A influência da Estratégia Saúde da Família no uso de serviços de saúde por crianças no Brasil: análise com escore de propensão dos dados da Pesquisa Nacional de Saúde. Cien Saude Colet. 2019;24(4):1495-505. http://dx.doi.org/10.1590/1413-81232018244.05522017. PMid:31066851.

25. Peixoto MRG, Ramos K, Martins KA, Schincaglia RM, Braudes-Silva LA. Insegurança alimentar na área de abrangência do Núcleo de Apoio à Saúde da Família em Itumbiara, Goiás. Epidemiol Serv Saude. 2014;23(2):327-36. http://dx.doi.org/10.5123/S1679-49742014000200014.

26. Souza BFNJ, Marin-Leon L, Camargo DFM, Segall-Corrêa AM. Demographic and socioeconomic conditions associated with food insecurity in households in Campinas, SP, Brazil. Rev Nutr. 2016;29(6):845-57. http:// dx.doi.org/10.1590/1678-98652016000600009.

27. Figueroa Pedraza D, Sales MC. (In)segurança alimentar e nutricional: desenvolvimento de um indicador do problema e experimentação em famílias da Paraíba, Brasil. Interações. 2014;15(1):79-88. http://dx.doi. org/10.1590/S1518-70122014000100008.

28. Gubert MB, Perez-Escamilla R. Insegurança alimentar grave municipal no Brasil em 2013. Cien Saude Colet. 2018;23(10):3433-44. http://dx.doi.org/10.1590/1413-812320182310.265120161. PMid:30365862.

29. Facchini LA, Nunes BP, Motta JVS, Tomasi E, Silva SM, Thumé E, et al. Insegurança alimentar no Nordeste e Sul do Brasil: magnitude, fatores associados e padrões de renda per capita para redução das iniquidades. Cad Saude Publica. 2014;30(1):161-74. http://dx.doi.org/10.1590/0102-311X00036013. PMid:24627023.

30. Morais DC, Dutra LV, Franceschini SCC, Priore SE. Insegurança alimentar e indicadores antropométricos, dietéticos e sociais em estudos brasileiros: uma revisão sistemática. Cien Saude Colet. 2014 maio;19(5):147588. http://dx.doi.org/10.1590/1413-81232014195.13012013. PMid:24897212.

31. Cotta RMM, Machado JC. Programa Bolsa Família e segurança alimentar e nutricional no Brasil: revisão crítica da literatura. Rev Panam Salud Publica. 2013 jan;33(1):54-60. http://dx.doi.org/10.1590/S102049892013000100008. PMid:23440158.

32. Poblacion AP, Cook JT, Marín-León L, Segall-Corrêa NA, Silveira JAC, Konstantyner T, et al. Food Insecurity and the Negative Impact on Brazilian Children's Health - Why Does Food Security Matter for Our Future Prosperity? Brazilian National Survey (PNDS 2006/07). Food Nutr Bull. 2016;37(4):585-98. http://dx.doi. org/10.1177/0379572116664167. PMid:27604621.

33. Sabóia RCB, Santos MM. Prevalence of food insecurity and associated factors in households covered by the Family Health Strategy in Teresina-PI, Brazil, 2012-2013. Epidemiol Serv Saude. 2015;24(3):749-58. http://dx.doi.org/10.5123/S1679-49742015000400017.

34. Wolf MR, Barros AA Fo. Estado nutricional dos beneficiários do Programa Bolsa Família no Brasil - uma revisão sistemática. Cien Saude Colet. 2014;19(5):1331-8. http://dx.doi.org/10.1590/1413-81232014195.05052013. PMid:24897198.

35. Ferreira HS, de Souza ME, Moura FA, Horta BL. Prevalência e fatores associados à Insegurança Alimentar e Nutricional em famílias dos municípios do norte de Alagoas, Brasil, 2010. Cien Saude Colet. 2014;19(5):153342. http://dx.doi.org/10.1590/1413-81232014195.06122013. PMid:24897218.

36. Ramalho AA, Martins FA, Koifman RJ. Food insecurity during the gestational period and factors associated with maternal and child health. J Nutr Health Food Eng. 2017;7(4):00245. http://dx.doi.org/10.15406/ jnhfe.2017.07.00245.

37. Marqui PA, Kuroyanagi FL, Foss MS, Dobre NR, Souza DN, Bittencourt WS, et al. Principais fatores da baixa adesão ao uso do ácido fólico. UNOPAR Cient Ciênc Biol Saúde. 2014;16(2):141-8. http://dx.doi. org/10.17921/2447-8938.2014v16n2p\%25p. 
38. Panigassi G, Segall-Corrêa AM, Marin-León L, Pérez-Escamilla R, Sampaio MFA, Maranha LK. Insegurança alimentar como indicador de iniqüidade: análise de inquérito populacional. Cad Saude Publica. 2008;24(10):2376-84. http://dx.doi.org/10.1590/S0102-311X2008001000018. PMid:18949239.

39. Linhares $A O$, Linhares RS, Cesar JA. Iniquidade na suplementação de sulfato ferroso entre gestantes no sul do Brasil. Rev Bras Epidemiol. 2017;20(4):650-60. http://dx.doi.org/10.1590/1980-5497201700040008. PMid:29267750.

40. Demétrio F, Teles-santos CAS, Santos DB. Food insecurity, prenatal care and other anemia determinants in pregnant women from the NISAMI Cohort, Brazil: hierarchical model concept. Rev Bras Ginecol Obstet. 2017;39(8):384-96. http://dx.doi.org/10.1055/s-0037-1604093. PMid:28666298. 